\title{
Mired in mosaicism: the perils of genome trivialization
}

\author{
David F. Albertini ${ }^{1}$
}

Published online: 28 October 2016

(C) Springer Science+Business Media New York 2016

\section{Commentary}

Some years ago, I had the chance to make an excursion from Bologna to Ravenna, experiencing at the suggestion of a close friend what was arguably one of the most astonishing collections of "mosaics" - in the artistic sense. Between the fifth and eigth centuries, Ravenna served first as the transitional stage for the Roman Empire eventually morphing into what became Byzantine Italy along the way to Constantinople - and as they say, the rest is history. Not so much for the elegance and beauty of artwork is Ravenna so impressionable to the serious or casual tourist as is the historical significance of how this art form became emblematic of the blending of cultures presaging a world poised to meld east and west in the grandest mosaic of all (http://whc.unesco.org/en/list/788). So much for the aesthetic side of mosaicism given the current level of discourse centered flatly in the middle of human ARTs past, present, and future!

Unsurprisingly and against this backdrop, JARG continues to contribute to what has been the ongoing debate on PGS/PGD by expanding our coverage on the matter of mosaicism well beyond the purview of ARTs and "formal" genetics. Unlike the state of affairs even 8 years ago, when Fritz stated that:

Capsule Few topics are engaging as much attention in the field of human ARTs these days as that of embryonic mosaicism in all its shapes and forms. With of main course offering locked onto the matter of utility and efficacy of PGS/PGD, as it was 8 years ago, perhaps the ongoing conversation should be cast in the light of how evolving concepts in basic science have failed to convey the building blocks and biological organization principles required to resolve questions of clinical import vested in by patients and physicians alike.

David F. Albertini

dalbertini@thechr.com

1 The Center for Human Reproduction, New York, NY, USA
"The concept of PGS is so logical and attractive that its efficacy was generally assumed and accepted without serious question, once its technical feasibility had been demonstrated" [1] more questions have been raised over the past year, strongly evidencing the importance of journals positioned deep within such subject matter to carry on the discourse at such a crucial point in the crossroads of reproduction and genetics broadly in the biomedical research enterprise. For those in our readership unfamiliar with the Fritz commentary, it is well worth taking a close look to remind ourselves that many of the same issues prompting a spectrum of emotionality today were firmly ensconced in a primordial technology (at least back then) seemingly poised to tackle that uniquely human nemesis -and premise- "...that the majority of failed implantations and early pregnancies reasonably may be attributed to embryo aneuploidy...."

Before delving further into the topic of mosaicism, we begin this month's coverage with two reviews that highlight distinctly non-genetic processes known to play directly into the success or failure of the human conceptus. The first review revisits a familiar subject evoked by the "seed or soil" metaphor re-emphasizing the delicate balance between embryo and endometrium that oversees initiation and maintenance of gestation through these earliest of stages in human development (What is the contribution of embryo-endometrial asynchrony to implantation failure? 10.1007/s10815-016-0773).

The second review looks more specifically into the embryo itself and brings to the surface a dimension of organization that is only now becoming recognized as a powerful force behind determining an embryo's viability and developmental potential. The subcortical maternal complex (SCMC) was first described in mouse embryos by Jurrien Dean and colleagues at the NIH. As the review from Bebbere et al. illustrates, evidence is accumulating which shows that many of the 
maternal gene products important for embryogenesis are stored, protected, and/or processed within a specialized compartment confined to the outer reaches of blastomeres [2]. Among the constituents of the SCMC are enzymes that are pivotal in genomic imprinting methylation and demethylation activities, one of which has been implicated in the etiology of recurrent hydatidiform moles [3]. These non-genetic, and in some cases clearly epigenetic, factors overseeing the very actions of gene products removed in space and time from when that gene was actively transcribed continue to confound our superficial reliance on "matters genetic" to explain the biological complexity of early human development.

A relevant example of the emergent conundrum imposed by perceived incompatibilities is illustrated on our cover this month. More than a mosaic, the paper from the Genetics of Male Fertility Group in Barcelona, Spain, draws attention to the importance of understanding genes and their position on chromosomes within the realm of chromosome territories, a fast evolving principle in explaining the influence of, in this case, Robertsonian translocations on male fertility [4].

Our issue continues with the thematic focus of mosaicism. First, we set the stage with two opinion pieces taking in sequence rearward and forward perspectives of where the issues come from and what may, or may not, be matters sure to remain on the podium for years to come. A series of original papers then attest to many of the issues currently at hand ranging from the impact of combined ARTs on decisions to transfer blastocysts, in the context of present day practices in the US [5], adoption and validation of PGS/PGD testing platforms, and an area of growing concern, variability in results within or between single centers offering such testing on a routine basis. As always, we hope you will join the conversation through original submissions or letters to the editor offering your insights and recommendations as a consensus in human ARTs is sought.

In the end, there are many reasons contributing to reproductive failure in humans well in excess of the attributions we make from counting chromosomes, or translocations, or ploidies. A host of underlying cellular forces distant in time and space from the birth of processed mRNAs are culpable for the aberrations in human embryo development (some visible, some not) that may validate iatrogenic weeding out or failure after transfer. And how can we overlook the complexities the progressive dialogue between mother and conceptus clearly obviating the need for a genetic contribution.

While debates will continue over sequencing technology, it is fair to ask whether we have lost sight of the principle of whole genome architecture (as noted above) while the rest of the biomedical enterprise has moved well beyond the concept of genes in refining their technology to acquire clinical specificity and validated utility removing all traces of uncertainty [6]. Perhaps, like the mosaics of Ravenna, there will someday be a sense of accomplishment and pride shared by scientists and clinicians over the multicultural achievements made on behalf of the ever-creasing number of couples desirous of parenthood.

\section{References}

1. Fritz MA. Perspectives on the efficacy and indications for preimplantation genetic screening: where are we now? Hum Reprod. 2008;23(12):2617-21.

2. Bebbere D, Masala L, Albertini DF, Ledda S. The subcortical maternal complex: multiple functions for one biological structure? J Assist Reprod Genet. 2016;15:416-25.

3. Akoury E, Zhang L, Ao A, Slim R. NLRP7 and KHDC3L, the two maternal-effect proteins responsible for recurrent hydatidiform moles, co-localize to the oocyte cytoskeleton. Hum Reprod. 2015;30(1):159-69.

4. Sole M, Blanco J, Valero O, Verges L, Vidal F, Sarrate Z. Altered bivalent positioning in metaphase I human spermatocytes from Robertsonian translocation carriers. J Assist Reprod Genet. 2016.

5. Scott Jr RT, Upham KM, Forman EJ, Hong KH, Scott KL, Taylor D, et al. Blastocyst biopsy with comprehensive chromosome screening and fresh embryo transfer significantly increases in vitro fertilization implantation and delivery rates: a randomized controlled trial. Fertil Steril. 2013;100(3):697-703.

6. Ay F, Noble WS. Analysis methods for studying the 3D architecture of the genome. Genome Biol. 2015;16:183. 